\title{
EL BINOMIO OCIO-FAMILIA DESDE EL ENFOQUE HUMANISTA: EL CASO DE LAS FAMILIAS DE LA CAJA DE COMPENSACIÓN FAMILIAR DEL HUILA
}

\author{
THE LEISURE-FAMILY BINOMIAL FROM THE HUMANISTIC APPROACH: \\ THE CASE OF FAMILIES FROM THE HUILA FAMILY BENEFIT FUND
}

O BINÔMIO LAZER-FAMÍLIA A PARTIR DA ABORDAGEM HUMANISTA: 0
CASO DAS FAMÍLIAS DO FUNDO DE COMPENSAÇÃO FAMILIAR DE HUILA

Ángel Miller Roa Cruz

Resumen

En este documento se presenta parte de los resultados de la investigación "El binomio ocio-familia desde el enfoque humanista: el caso de las familias de la Caja de Compensación Familiar del Huila”. De tal manera, se ofrece al lector una guía de algunos de los contenidos abordados en dicha investigación, teniendo como referentes su marco general, su justificación y la pertinencia de sus propuestas, los objetivos y la metodología utilizada para llevarlos a cabo. Posteriormente, se hace una aproximación teórica del ocio, por lo que se aborda el tema de las "Transformaciones y avances hacia el ocio humanista". De este apartado se desprenden otras temáticas: el ocio como factor de desarrollo humano; el ocio humanista como alternativa: sentido e importancia. El siguiente apartado se llama "El binomio ocio y familia: desde la teoría a la realidad", del cual se abordará la temática "El rol de la familia en el ocio familiar". Un cuarto apartado llamado "Diagnóstico" el cual condensa, a través de una matriz Dafo, algunos de los resultados de la investigación. Y finalmente algunas conclusiones.

Palabras clave: ocio; humanismo; autotelismo; tiempo libre; recreación; cajas de compensación familiar

\section{Abstract}

This document presents part of the results of the research, "The Leisure-Family Binomial from the Humanistic Approach: The Case of Families from the Huila Family Benefit Fund." As such, it offers the reader a guide to some of the contents addressed in this research, using as benchmarks its general framework, its justification and the pertinence of its proposals, the objectives, and the methodology used to carry them out. Then, a theoretical approach to leisure is made, thus addressing the subject of "Transformations and progress towards humanistic leisure." Other topics emerge from this section: leisure as a factor of human development; humanistic leisure as an alternative: meaning and importance. The next section is called "The leisure and family binomial: from theory to reality," which addresses the issue of "The role of family in family leisure." A fourth section, "Diagnosis," condenses, through a swot matrix, some of the results of the research. The last section presents the conclusions.

Keywords: leisure; humanism; autotelism; free time; recreation; Family Compensation Funds 


\section{Resumo}

Este artigo apresenta parte dos resultados da investigação " O binômio lazer - família a partir da abordagem humanista: o caso das famílias do Fundo de Compensação Familiar de Huila”. Assim, oferecemos ao leitor orientações para alguns dos conteúdos abordados nesta pesquisa, tendo como referência o marco geral, a justificação e relevância de suas propostas, os objetivos e a metodologia utilizada para realizá-los. Posteriormente, realizamos uma abordagem teórica do lazer, na que incluímos o tema "mudança e progresso para o lazer humanista". A partir desta seção emergem outros tópicos: o lazer como fator de desenvolvimento humano; o lazer humanista como alternativa: sentido e importância. A partir da seguinte seção, intitulada " 0 binômio lazer-família: da teoria à realidade”, abordamos o tema "0 papel da família no lazer familiar”. A quarta seção, "Diagnóstico", dispõe, através de uma análise swot, alguns dos resultados da investigação. Finalmente, apresentamos algumas conclusões.

Palavras-chave: lazer; humanismo; autotelismo; tempo livre; recreação; Fundos de Compensação Familiar 


\section{INTRODUCCIÓN}

Las consecuencias del ocio, el tiempo libre, y el trabajo en el ser humano se manifiestan en la sociedad. La aparición del estrés deteriora la salud y provocan agotamiento físico y mental en la individualidad de los seres, por lo que se insertan en el conjunto de necesidades que los Estados modernos han procurado satisfacer a lo largo de la historia, creando estrategias e instituciones que generan un ámbito propicio para garantizar la seguridad social de las personas. Si bien es cierto que la recreación y el sano esparcimiento se enmarcan en ese conjunto de necesidades básicas aún insatisfechas en buena parte de los países latinoamericanos, los Estados sociales las han reconocido como un derecho fundamental para el desarrollo armónico de los ciudadanos, como es el caso de Colombia:

El ejercicio del deporte, sus manifestaciones recreativas, competitivas y autóctonas tienen como función la formación integral de las personas, preservar y desarrollar una mejor salud en el ser humano [...] forman parte de la educación y constituyen gasto público social. (Constitución Política de Colombia, 1991, art. 52).

Las cajas de compensación cumplen funciones de amplia variedad relacionadas con el desarrollo social y su papel, que conjuga bienestar, calidad de vida y responsabilidad social.

En Latinoamérica, estas organizaciones desempeñan, entre otros, un papel que es parte de su responsabilidad social: generar alternativas que contribuyan al bienestar de sus afiliados partiendo del reconocimiento de la recreación sana, el disfrute del ocio y el tiempo libre como oportunidades para el encuentro, generar esparcimiento, desarrollar creatividad y procurar descanso. Todo ello favorece y contribuye a la convivencia pacífica, la tolerancia y el desarrollo integral de las personas y comunidades.

Por esta razón, no se puede olvidar que su naturaleza, incluyendo la filosofía institucional que las enmarca, simboliza oleadas de diversión, sano esparcimiento y tiempo libre en escenarios de ocio y recreación que hacen factible y fundamental el desarrollo integral del ser humano.

Se trata, entonces, de una "reivindicación utópica de un tiempo libre equilibrado, cuantitativamente, con el trabajo y el sueño. Tiempo libre como derecho social de todo ser humano" (Cuenca, 2009, p. 14). Producto de estas conquistas sociales, todo el mundo puede llegar a tener tiempo libre pero no siempre una experiencia de ocio; entonces, tener un trabajo es parcialmente garante de tener una mejor calidad de vida, así como tener tiempo libre es parcialmente garante de vivir una experiencia de ocio. Es, entonces, cuando se advierte la necesidad de educación del ocio como estrategia adecuada para garantizar su optimización y potencialidades como factor de desarrollo humano.

\section{CONTENIDO}

Los referentes de este documento están soportados con estudios sobre ocio, como los descritos en las producciones de Bolaño (1999) y Cabanzo (2009); aunque con pesar ha de describirse la ausencia de esta clase de investigaciones en la región, razón que hace más insistente y relevante la formulación de esta clase de estudios.

Es de señalar que el principal centro que brinda recreación y bienestar en el departamento del Huila es la Caja de Compensación Familiar, por lo que su misión está orientada al liderazgo de programas de intervención social que generan inclusión y bienestar en afiliados y comunidad en general, pero que, pese a su liderazgo, no cuenta con una política de ocio que fortalezca las bases de crecimiento y desarrollo a escala.

En este sentido, a través del contacto que se tiene con estos dos universos, el empresarial y el académico, ambos conectados con los temas de ocio, recreación y tiempo libre, imbricados en las dinámicas culturales de una ciudad pequeña como Neiva, también constituye un problema la carencia de espacios y programas en la ciudad que promuevan el ocio y sus variadas manifestaciones: música, literatura, danza, teatro, cine, etc., entre otras actividades.

Desde esta perspectiva, el aporte de esta investigación es ampliar el conocimiento sobre la experiencia de ocio de las familias beneficiarias de la Caja de Compensación Familiar. Así, conocer la experiencia de ocio en familia permitirá comprender las dinámicas de interacción de las familias durante el tiempo libre. Por ello, el objetivo general de esta 
investigación fue diseñar una propuesta de ocio para la Caja de Compensación Familiar en la ciudad de Neiva (Colombia) que, desde una perspectiva humanista y partiendo de la caracterización y comprensión de las experiencias de ocio de sus familias beneficiarias, contribuya a hacer de su ocio un factor de desarrollo personal y social

Por otra parte, este tipo de estudios en torno al ocio facilitan la construcción de referentes para la configuración de políticas institucionales orientadas a mejorar la contribución de los programas de ocio al bienestar de las familias. Finalmente, este conocimiento permite avanzar en Colombia hacia la elaboración de un modelo de ocio innovador, enfocado en la consolidación del contexto familiar como el primer espacio básico de convivencia.

En este contexto, el presente estudio tiene un enfoque metodológico mixto. Así pues, se utilizan instrumentos de recolección propios de la investigación cuantitativa y cualitativa para el caso de las familias y los administrativos y directivos, respectivamente: talleres, encuesta y entrevista. La encuesta representa un instrumento clave como herramienta que permite adquirir información en pos de dar respuestas a los objetivos e interrogantes planteados. De igual forma, desde el paradigma cualitativo, los talleres y entrevistas semiestructuradas en profundidad son importantes para percibir de manera profunda las características y experiencias del ocio en las familias beneficiarias de Comfamiliar Huila.

La unidad de análisis del presente estudio la constituyen los directivos de Comfamiliar y la familia neivana, afiliada a la Caja de Compensación Familiar del Huila, clasificada en las categorías A, B y C, de acuerdo con el nivel de ingresos. De dicha unidad de análisis, inicialmente, se asumieron unos estudios exploratorios y se considera el taller como una estrategia pertinente para conocer qué idea tienen los miembros de las familias sobre estos conceptos y luego, en este sentido, diseñar la encuesta.

Posteriormente, en un siguiente momento se establece la implementación de la encuesta con los afiliados de Comfamiliar, cuyas preguntas apuntaban a dar respuesta a los objetivos específicos planteados. Luego, la entrevista, que se constituye en la herramienta elegida para recolectar la información de los directivos y administrativos de Comfamiliar.
El último momento es la realización de proyectos de educación en ocio, amparados en el modelo de intervención de la Universidad de Deusto, UD. Desde este modelo, se plantea la necesidad de diferenciar entre educación del ocio a nivel personal (para nuestro caso, fueron los directivos y funcionarios de Comfamiliar), a nivel comunitario (los afiliados a Comfamiliar y su grupo familiar) y de profesionales del ocio (investigador y equipo de trabajo).

El soporte teórico se confrontó con la revisión sistemática desde la literatura científica, iniciada desde la importancia del ocio en la familia, la cual se advierte como pieza clave entre los agentes educativos implicados en las experiencias de ocio. De igual forma, se hace énfasis en la manera como dicho núcleo familiar recibe el influjo del contexto social, de la cultura, y, también, del mundo imaginario que se teje, sobre todo, en la mente de los niños y adolescentes, y aún más en quienes fijan su interés en los caminos del arte.

De esta manera, conceptos como el de ocio y familia, en el contexto de las cajas de compensación familiar en Colombia, parten de la creación de las cajas de compensación familiar en Colombia y en Latinoamérica, y resaltan la idea que estas surgieron como una necesidad social sin atenerse a reglamentaciones o leyes de ningún tipo. Seguidamente, se aborda el surgimiento de las cajas de compensación familiar (CCF), en Colombia, su desarrollo jurídico, reconociendo el ocio como un derecho fundamental y las formas en las que deben ser aplicados los recursos que aportan los empresarios para contribuir al logro de la política de seguridad social.

\section{TRANSFORMACIONES Y AVANCES HACIA EL OCIO HUMANISTA}

Si bien el ocio ha sido objeto de estudio por parte de las ciencias sociales desde hace algunas décadas, $\mathrm{y}$, por parte de los académicos que se han ocupado de dicho concepto, no hay duda respecto a la importancia que reviste como fenómeno científico y también social, en cuanto su papel para la mejora de la calidad de vida de la ciudadanía (Monteagudo, 2008). Sin embargo, el ocio presenta, aun en algunos contextos geográficos y grupos de población, connotaciones peyorativas, relacionadas con la pérdida de tiempo, asumiendo que con él no se realiza ninguna actividad favorable para la vida. En la comunidad científica 
tampoco existe consenso absoluto en torno al concepto de ocio, tiempo libre, y recreación.

Este hecho obstaculiza el reconocimiento universal del ocio como idea e ideal, pero sobre todo su papel como motor de desarrollo social y humano. Por eso, no en vano la insistencia de seguir analizando y aproximándonos teóricamente al ocio, a partir de los contextos particulares de cada país, sus culturas, de la naturaleza de las instituciones que operan en torno al ocio, interpretaciones y formas de experimentarlo.

La ineludible relación entre ocio, desarrollo humano y humanismo nos lleva también a la necesidad de pensar un ocio humanista como alternativa y forma de mediación en un escenario dominado por el capitalismo extremo y sus desmedidas formas de consumo; así pues, desde esta tesis, se considera necesario no solo pensar la idea e ideal de ocio, sino también sentar las bases para la construcción colectiva de sus sentidos, amparados por un enfoque humanista.

De esta manera, el primer aspecto que se aborda es el de las relaciones entre ocio, tiempo libre y recreación, conceptos que han sido ampliamente debatidos y cuyos acuerdos, hoy, nos develan un horizonte más claro respecto a sus diferencias, puntos de encuentro y prospectivas, ya sea desde los linderos de la pedagogía o desde contextos empresariales donde se operan estas formas de bienestar.

\section{¿POR QUÉ REFLEXIONAR SOBRE OCIO, TIEMPO LIBRE Y RECREACIÓN?}

Diversos autores consideran el ocio una necesidad, pero hay quienes aproximan la experiencia del ocio a un proceso de bienestar e incremento de la calidad de vida; por otro lado, enmarcan tiempo libre y recreación con dinámicas de consumo, analizadas en una economía capitalista en la que el dinero se impone como lenguaje universal, en donde las industrias del entretenimiento forman parte del conjunto de entes homogenizadores de necesidades, deseos e ilusiones. Ocio, tiempo libre y recreación: ¿necesidad de tiempo libre para el consumo? Sin lugar a dudas la forma en que se asuma y experimente el ocio, a nivel personal o colectivo, determinará la respuesta del anterior interrogante. Pasa igual con la forma en que se asuma el tiempo libre y recreación.
Los teóricos Cuenca, Aguilar, y Ortega, (2010), advierten que incluso el hecho de tener tiempo libre no garantiza un experiencia de ocio y que este último, a su vez, tiene lugar en la vida de una persona como consecuencia de una formación, es decir, cultivar aquellas prácticas en las cuales cada quien define buena parte de su identidad, con la particularidad de que, a través del ocio y de su noción lúdica, la persona disfruta en el ejercicio de ese autoconstructo (Cuenca, 2009).

\section{OCIO Y TIEMPO LIBRE}

En este apartado se hace énfasis en la noción de tiempo libre y tiempo de trabajo, producto de los procesos de industrialización, especialmente en países europeos, donde múltiples luchas sociales y sindicales, animadas por intelectuales como Beltrán Russel o Paul Lafargue, han abierto el camino a la posibilidad de resignificar la idea de desarrollo y, sobre todo, de bienestar. Se trata entonces de una "reivindicación utópica de un tiempo libre equilibrado, cuantitativamente, con el trabajo y el sueño. Tiempo libre como derecho social de todo ser humano" (Cuenca, 2009, p. 13). Producto de estas conquistas sociales, todo el mundo puede llegar a tener tiempo libre, pero no siempre una experiencia de ocio; entonces, tener un trabajo es parcialmente garante de tener una mejor calidad de vida, así como tener tiempo libre es parcialmente garante de vivir una experiencia de ocio. ¿Por qué razón? ¿Qué se entiende por estos dos conceptos?

Autores como Dumazedier, J. (1969) han resaltado que el ocio generalmente se ha definido por sus funciones, y describe estas como la relajación, el entretenimiento y el desarrollo personal, o los tres grupos generales estudiados por Sue (1982): funciones psicológicas (descanso, diversión y desarrollo), funciones sociales (sociabilidad, simbolismo o distinción personal), y funciones terapéuticas y económicas, como complemento del proceso de producción y generadoras de consumo.

En este sentido, Rodríguez (1992) asegura que debe intentarse una aproximación al ocio en sí mismo. Analizarlo como la negación del tiempo. Así pues, para Cuenca, las experiencias de ocio transcurren en el tiempo, pero a la vez también son y se califican gracias al tiempo: 
El tiempo incide en la vivencia de ocio no solo desde un punto de vista histórico y cuantificador, sino marcando tonalidades teñidas por las vivencias del pasado y la esperanza de un tiempo mejor. Nuestros ocios no son solo presente, sino itinerarios de vida que hablan de comienzos, desarrollos, momentos cumbre y declive, marcados por un tiempo propio. (Cuenca, 2009, p. 13)

En este contexto, se tienen varios elementos: tiempo, que tiene diferentes matices - tiempo de trabajo, tiempo disponible (no necesariamente para el ocio), tiempo libre (que pude ser destinado para el ocio pero puede que la experiencia ociosa no tenga lugar) -; y, por otro lado, el ocio. El tiempo se advierte, entonces, como una posibilidad a la que, en la sociedad actual, se le atribuye sentido. Así, la posibilidad del tiempo libre, hoy en día un derecho humano, se refiere a una forma de calcular una determinada clase de tiempo (De Grazzia, 1982), sea que dicho cálculo temporal se asigne por la misma persona, por las instituciones a las que pertenece -empresa, familia, juntas de acción comunal, sindicatos; y en las que ejerce diferentes roles: padre de familia, hijo, líder social o político, esposo, etc.-, o por el azar, condicionado a los otros tiempos y sus dinámicas. Se puede entender, entonces, el tiempo libre como un tiempo exento de obligaciones que la persona tiene para sí misma, para hacer aquello que desea, o que no desea, pero podría desear. El tiempo libre es sin duda una posibilidad.

Ahora bien, este tiempo libre se constituye en posibilidad de tiempo para el ocio cuando hay una disposición para afrontarlo de forma autónoma y satisfactoria, lo cual se traduce en "la gestión de actividades escogidas libremente con el fin de disfrutar, descansar, sentirse realizado" (Muñoz, 2002, párr. 6).

De esta manera, si el tiempo libre es posibilidad, el ocio se constituye en una forma de dar sentido a esa posibilidad. Al respecto, Cuenca y Aguilar aseguran que "la vivencia plena del ocio se produce cuando se lleva a cabo como experiencia completa y con sentido, es decir, cuando existe un proceso con inicio, nudo y desenlace" (Cuenca, M., Aguilar, E. y Ortega, C. 2010, p. 16), lo que nos lleva a relacionar el proceso del inicio con el hecho de pensar, desear y organizar una actividad; el nudo, con su realización; y el desenlace, el acto de recordarla con satisfacción.
El ocio es, entonces, una especie de "relato" autotélico; dicho relato, por más que se tenga tiempo libre para llevarse a cabo, puede sencillamente no hacerse y de esta manera la variable tiempo quedaría vacía; o llena de alguna otra actividad que, al no generar satisfacción, no sería ociosa. Es por esto que el tiempo libre no puede ser sinónimo de ocio.

Neulinger (1980) entendía que el trabajo también podía ser ocio. Advertía que la percepción de libertad de la persona, junto con la motivación que algo le pudiera generar, constituyen las condiciones esenciales para hablar de ocio.

Un ejemplo que podría soportar dicha premisa radica en las personas cuyo oficio es a su vez su pasión: vive de eso, y disfruta haciéndolo, como algunos músicos, escritores de ficción, actores, fotógrafos, etc. Incluso, siendo un poco más osados con este enfoque, cabe una pregunta: ¿un oficio cualquiera, que a su vez cumpla con las condiciones de lo que hoy reconocemos como trabajo, podría ser ocio si cumple también con las condiciones de este?

Tal vez una de las condiciones esenciales que definen el trabajo radica en que se trata de una actividad, sea de origen manual o intelectual, que se realiza a cambio de una compensación económica por las labores hechas.

Si volvemos a los casos más evidentes, el de los artistas cuyo oficio es a su vez su pasión, motivación, lo hacen de manera libre y reciben compensación económica por ello.

\section{OCIO HUMANISTA VS. VALORES}

Se necesita contar con una formación humanista para el ocio sobre todos los ámbitos familiares y educativos para con ella identificar características que vayan más allá de la recreación y del tiempo libre y poder aportar sentido a la actividad. Formarse para el ocio humanista es aprender a establecer vínculos, aprender a valorar desde la subjetividad, prácticas y medios que ofrecen la sociedad y la cultura.

Desde este entorno, Cuenca (2005) afirma que "el ocio es, sobre todo, el ámbito en el que se demuestra la elección de valores" (p. 43). Surge, entonces, una pregunta: ¿qué valorar para lograr incorporar en nuestras vidas un ocio humanista? 
Pero antes de resolver este interrogante, es necesario abordar específicamente el concepto de valores. Para González y Marquínez, los valores son "determinadas maneras concretas de apreciar ciertos aspectos importantes en la vida humana, por parte de los individuos que pertenecen a un determinado grupo cultural" (González y Marquínez, 2007, p. 32). Y es precisamente esta, la cultura, la que moldea las maneras de apreciar por parte del individuo, en y con el grupo social, las cosas importantes de la vida como pueden ser la vida misma, el amor, el trabajo, la fiesta, la familia, la libertad, el tiempo, entre otros.

\section{RESULTADOS}

Obedecen a la información recolectada de la unidad de análisis, la familia y los administrativos y directivos de Comfamiliar. En la primera parte se enseñan las reflexiones que se obtuvieron a través de los talleres a un grupo de familias beneficiarias de Comfamiliar, respecto a los conceptos y temáticas "tiempo libre y ocio", "importancia del ocio en la integración familiar" y "expectativas para satisfacer la necesidad de ocio".

Posteriormente, se presentan las concepciones de ocio de las familias afiliadas a la empresa, obtenidas a través de la encuesta y agrupadas mediante categorías: composición del núcleo familiar, descripción del ocio, tiempo libre en familia, tiempo libre en Comfamiliar y recomendaciones. La segunda parte comprende el análisis de las entrevistas realizadas a los cinco directivos y a los cuatro administradores de los centros recreativos de Comfamiliar Huila con experiencia mínima de dos años en el cargo cuyas preguntas estaban encaminadas a conocer su concepción de ocio, políticas de ocio en Comfamiliar y estrategias de Comfamiliar encaminadas a crear conciencia en los afiliados sobre el uso adecuado del tiempo libre.

Se diseña, de igual forma, un diagnóstico amparado en resultados de la encuesta y en las variables que aborda: a) concepción de ocio, su nivel de importancia y expectativas; b) actividades de ocio en familia; c) valores que se tejen en las actividades de ocio; d) conocimiento que tienen las familias sobre las estrategias de Comfamiliar encaminadas a crear conciencia sobre el uso adecuado del tiempo libre; e) apropiación de los espacios de ocio de Comfamiliar por parte de las familias; f) el nivel de participación e interés de las familias afiliadas en las actividades de ocio programadas por Comfamiliar; $y$ de igual forma, teniendo en cuenta el análisis de las entrevistas realizadas a los administrativos de la empresa, se advierten algunas conclusiones e ideas en cada una de ellas, de cara a la propuesta de ocio.

La población sobre la cual se hizo aplicación de la encuesta estuvo compuesta por 8900 empresas afiliadas, las cuales incorporan 102193 trabajadores con un total de 176938 personas afiliadas beneficiarias en todo el departamento del Huila, para un gran total de 279131 entre afiliados y beneficiarios.

Las cajas de compensación involucradas para el departamento del Huila se preestablecieron en los municipios con las respectivas agencias en Neiva (83 259 P. trabajadora), Garzón (6702 P. trabajadora), Pitalito (9203) y La plata (3029 P. trabajadora).

La muestra la conformaron 364 familias definidas mediante muestreo probabilístico simple por conglomerados identificando las categorías que establece la caja para la respectiva afiliación $A, B, C$, de las cuales 247 pertenecen a la categoría A, 78 a la B y 39 a la C.

Los resultados obtenidos se apoyan en estadísticas descriptivas y relacionales. Para la relación entre variables se aplicaron pruebas de hipótesis apoyadas en una distribución chi cuadrado, con niveles de significancia del $5 \%$. Según Sampieri, Fernández y Baptista, (2010), la distribución chi cuadrado es una distribución de probabilidad que posibilita el análisis relacional bivariado (relación entre dos variables), planteando dos hipótesis: la hipótesis nula o de independencia y la hipótesis alterna o de dependencia entre las variables, se genera la tabla de contingencia y se calculan los valores de significación mediante el paquete estadístico SPSS. Estos valores generan resultados de aceptación o rechazo sobre la hipótesis nula.

Los resultados de la encuesta se presentan agrupando los ítems mediante las categorías: composición del núcleo familiar, descripción del ocio, tiempo libre en familia, tiempo libre en Comfamiliar y recomendaciones.

En lo que respecta a la percepción de ocio de las familias a través de los talleres, se dieron evidencias de poca relación entre integrantes de la familia con el concepto de ocio y cómo se asimilaba de mejor manera el concepto de tiempo libre; se amplió en la 
socialización de las conclusiones y fueron incorporados en la encuesta otros temas fundamentales como los valores y el conocimiento e interés de las familias en las actividades de ocio que ofrece Comfamiliar.

Se realizó, de igual manera, un análisis relacional con variables de percepción de ocio de las familias encuestadas, valores que fomenta el ocio, actividades lúdicas, entre otras, y la variable categoría, la cual hace referencia a la clasificación de las familias de la Caja de Compensación Familiar del Huila en categoría A, B y C (Sampieri, et. al, 2010)

Para cada una de estas pruebas se considera el nivel de confianza que se desea que tengan los cálculos de la prueba; es decir, si se quiere tener un nivel de confianza del $95 \%$, el máximo error es del $5 \%=0,05$, lo cual corresponde al complemento porcentual de la confianza. El paquete sPSS genera dos tablas; la primera de ellas corresponde a la tabla de contingencia, en ella aparecen las variables seleccionadas con sus respectivas frecuencias y porcentajes. La segunda tabla corresponde a la prueba de chi cuadrado de Pearson y en ella aparecen los resultados de las pruebas: valor del chi cuadrado, razón de verosimilitud y los valores de significación (última columna). Elegimos el menor de estos valores.

La prueba de independencia del chi cuadrado parte de la hipótesis que las variables son independientes; es decir, que no existe ninguna relación entre ellas y por lo tanto ninguna ejerce influencia sobre la otra. El objetivo de esta prueba es comprobar la hipótesis nula mediante el nivel de significación, por lo que si el valor de la significación (el mínimo de la última columna) es mayor o igual que 0,05 , se acepta la hipótesis, pero si es menor se rechaza.

Para calcular el valor de significación, el paquete estadístico sPss calcula un valor llamado chi cuadrado midiendo la diferencia global entre los recuentos de casilla observados y los recuentos esperados. Cuanto mayor sea el valor de chi cuadrado, mayor será la diferencia entre los recuentos observados y esperados, lo que nos indica que mayor es la relación entre las variables.

El valor de significación corresponde a la probabilidad de que una muestra aleatoria, extraída de chi cuadrado, nos dé como resultado un valor superior al valor crítico bajo el cual se encuentra el $95 \%$, es decir, es la probabilidad que los datos de una muestra aleatoria extraída de las dos variables sean independientes.

Entre los resultados que se advierten, se tiene que para las familias el ocio hace referencia al tiempo libre que las personas utilizan para relajarse y distraerse de sus ocupaciones rutinarias; para la mayoría de los encuestados, es importante para sus vidas porque constituye una forma de entretenimiento familiar. En cuanto a los valores, el que más resalta es el de la alegría, lo cual indica la relación implícita que advierten los encuestados entre el ocio con la lúdica.

\section{CONCLUSIONES}

En Colombia, jurídicamente, a partir de la Asamblea Nacional Constituyente convocada en 1990, se fijaron políticas claras y criterios específicos en materia de recreación y deporte, con lo que se abrió la puerta a la legitimación del ocio como derecho, al menos en el papel. Así pues, ya en la Constitución del 91, el título II dedica un artículo especial, el 52, para establecer como derecho fundamental la recreación para todas las personas, sin distingo alguno; de igual forma, compromete al Estado a fomentar esta actividad, además de responsabilizarlo por su fomento.

Este aval jurídico ha pasado por un proceso lento $y$ largo para llegar a tener arraigo en la cultura, esto producto de diversos factores: las prioridades de los gobiernos se han enfocado en la guerra contra los grupos alzados en armas, de derecha e izquierda, guerras que son a su vez producto de la desigualdad e inequidad de las riquezas del país, lo que a su vez sigue alimentando fenómenos como el desplazamiento forzado de colombianos, en su mayoría campesinos, para quienes el ocio y la recreación constituyen un lujo prescindible respecto a otras necesidades (la alimentación, el techo, la misma vida).

Sin embargo, aun en este ámbito social adverso, las prácticas de ocio, entendidas como experiencias significativas en torno a diferentes tipos de actividades, trascienden la carencia de programas recreativos, o de espacios para ello, y dan vía libre a la creatividad y a la puesta en marcha del gozo a través de cualquier práctica u objeto (como sentarse en una esquina a hablar con los amigos, o recorrer las calles de la ciudad, en grupo, a ciertas horas, ver o escuchar por 
radio un partido de fútbol de su equipo favorito), lo cual, en ocasiones, da a luz culturas juveniles y nuevas formas de concebir el ocio.

Esto lo han entendido muy bien los científicos sociales en Colombia, sobre todo en ciudades como Medellín, Bogotá, Cali, y ahora Neiva, en donde se han realizado investigaciones respecto a las formas de ocio, recreación y tiempo libre en sus diferentes regiones.

Vale la pena resaltar que, en buena parte de estas investigaciones, el aspecto lúdico, como temática central y propia del objeto de estudio de la recreación, no se encuentra lo suficientemente consolidado como objeto propio de interés investigativo por parte de estudiantes y docentes. Esto se advierte sobre todo en investigaciones realizadas en contextos sociales vulnerables, en donde las prácticas de ocio se asumen y se analizan más como herramienta para sublimar conductas socioemocionales adversas que como práctica autotélica.

Ahora bien, se debe señalar que esta investigación aborda el tema del ocio tomando como eje central de su unidad de análisis a la familia, específicamente las familias de la Caja de Compensación Familiar en la ciudad de Neiva; en tal sentido, se puede concluir que un rasgo característico de la familia colombiana y de su impacto sobre la unidad familiar, es la coexistencia de una diversidad de tipologías, producto del ajuste a los contextos urbanos o rurales, o a las condiciones socioeconómicas de los distintos estratos sociales; de igual forma, se constituye en rasgo común la inestabilidad afectiva y cohabitacional en las relaciones de pareja y de familia, es decir, la propensión a la ruptura y a las separaciones conyugales. Lo anterior, debido a diferentes causas como conflictos entre los distintos miembros; incluso el mismo conflicto armado que lleva más de 50 años ha incidido en la ruptura familiar, sobre todo en zonas rurales, pues muchos padres han muerto en medio de la guerra.

Así, la situación de ruptura o divorcio, y los procesos previos o posteriores, tienen consecuencias económicas, legales y afectivas para cada uno de los miembros de la familia nuclear, del sistema familiar más amplio y de la sociedad.

En este sentido, se puede concluir que las prácticas de ocio como mecanismo de liberación y gozo cons- tituyen un aspecto importante en las vidas de los colombianos, como individuos, como grupo y, sobre todo, como familia; ya se puedo comprobar, para el caso de las familias pertenecientes a la Caja de Compensación Familiar (CCF), a través de las encuestas realizadas en esta investigación, que la principal actividad de ocio era "compartir en familia"; por otro lado, aun cuando esta última ha sufrido profundos cambios durante las últimas décadas, los cuales han repercutido en los roles que sus miembros desempeñan respecto a temas como la educación, el trabajo y las relaciones de poder, se advierte entre la dupla ocio-familia un tercer actor conocido hoy como las cajas de compensación familiar. Estas desempeñan un papel determinante en los usos del tiempo libre de las familias, como es el caso de Comfamiliar.

\section{REFERENCIAS}

Bolaño, T. (1999). Estado actual de la investigación en ocio, tiempo libre y recreación. I Simposio de Investigación y Formación en Recreación. Vicepresidencia de la República, Coldeportes, Funlibre. Recuperado de http:// www.redcreacion.org/documentos/simposio1if/TBolano.htm

Cabanzo, C. (2009). Ocio y tiempo libre: una aproximación a sus representaciones y percepciones en la Universidad Pedagógica Nacional. Bogotá: Universidad Pedagógica Nacional.

Colombia, Asamblea Nacional Constituyente. Constitución Política de Colombia (6 de julio de 1991).

Cuenca, M. (2005). El ocio, un ámbito de cohesión familiar [Documento de trabajo n. 1]. Bilbao: Universidad de Deusto.

Cuenca, M. (2009). Tiempo ejemplar: el ocio y los mundos vivenciales. En M. Cuenca,.El tiempo de Ocio: transformaciones y riesgos en la sociedad apresurada. Documentos de ocio 36 .

Cuenca, M., Aguilar, E., y Ortega, C. (2010).Ocio para innovar. Documentos de estudio de Ocio, 42. Bilbao: Universidad de Deusto.

De Grazzia, S. (1982). Tiempo, trabajo y Ocio. Ciudad de México: Fondo de Cultura económica.

Dumazedier, J. (1969). Hacia una civilización del ocio. Barcelona: Editorial Estela.

Gonzales, L.J. y Marquínez, G. (2007). Valores éticos para la convivencia. Bogotá: Editorial El búho. 
Monteagudo, M. (2008). La experiencia de ocio: una mirada científica desde los estudios de ocio. Documentos de estudios de ocio, 35. Bilbao: Universidad de Deusto.

Muñoz, G. y Marín, M. (2002). Secretos de Mutantes. Bogotá: Universidad Central, Siglo del Hombre Editores.

Neulinger, J. (1980). Thepsychology of leisure. Springfield: charles Cthoma.
Rodríguez J. C. (1992). Tiempo y Ocio. Crítica de la Economía del Trabajo. Bogotá: Universidad Externado de Colombia.

Sampieri, H., Fernández, C., y Baptista, P. (2010). Metodología de la investigación. 5 Ed. Ciudad de México: McGraw-Hill.

Sue, R. (1982). El ocio. Fondo de cultura económica. México. 